\title{
Technologies of humility
}

\author{
Researchers and policy-makers need ways for accommodating the partiality of scientific \\ knowledge and for acting under the inevitable uncertainty it holds.
}

\section{Sheila Jasanoff}

The great mystery of modernity is that we think of certainty as an attainable state. Uncertainty has become the threat to collective action, the disease that knowledge must cure. It is the condition that poses cruel dilemmas for decision-makers; that must be reduced at any cost; that is tamed with scenarios and assessments; and that feeds the frenzy for new knowledge, much of it scientific.

For a long time we accepted lack of certainty as humankind's natural lot. What has happened to reverse that presumption? Perhaps it is the spread of binary thinking that frames the future in terms of determinate choices between knowable options. Boolean algebra and digital logics are not only built into our computers, mobile phones and other information and communication technologies, they dominate the framing of social problems and the options for dealing with them.

Thus, statistics offers a choice between Type 1 and Type 2 errors. The first lead to false positives that promote too much risk avoidance, the second to false negatives that keep us from acting when we ought. Implicitly, error follows a binary trail. Philosophy casts moral dilemmas as trolley problems, in which possible solutions are represented as choices encountered at forks in the track. One option is to let the trolley run its course and let five people die; the other is to throw a fat man on to the track, diverting the trolley and killing only one person. Which is the moral choice? Decision theory adopts one way of thinking and reasoning as rational; all others are biased by definition and need to be explained away as aberrations of human cognition. Even the concept of the win-win solution assumes, in binary logic, that for each party to a game, winning and losing are the only options.

Life, as we know from experience, seldom unfolds in binaries. We rarely confront Hamlet's choice - to act or not to act. There are always added considerations. Which action is best, by what criteria, how soon, with what provisos, at what cost and with what allowance for error? Even the half-mad prince recognized that secondorder consequences might complicate his first-order decision: to be or not to be.

Real problems in the real world are

infinitely complex, and for any given problem, science offers only part of the picture. Climate scientists can tell us with high certainty that human activities are raising Earth's mean surface temperature, that extreme weather events will occur, and that melting ice caps will cause abrupt

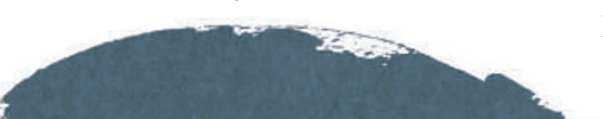

